\title{
A synchronizing second order sliding mode control applied to decentralized time delayed multi-agent robotic systems: Stability Proof
}

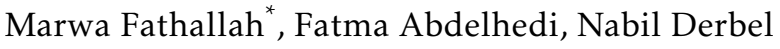

University of Sfax, Electrical Engineer, Sfax Engineering School, 3000, Tunisia

\begin{abstract}
A B S T R A C T
This study investigates the synchronization issue of multiple robot manipulators in the presence of time delay. Since several previous works based on synchronization task neglect the communication delay, in this paper we develop a finite time stability based on a Lyaponov functional for synchronization of a networked robotic system where time delay exists during the communication between robots. To this effect, we consider a second order sliding mode control (SMC) combined with the cross coupling concept in order to ensure the position synchronization of networked robot manipulators. Furthermore, the stability of the proposed controller with communication's delay has been proved. Simulation results illustrate satisfactory performances which prove the efficiency of the proposed approach.
\end{abstract}

\section{Introduction}

Over the past few decades, an increasing interest has been noticed on the interconnected systems in several fields of research $[1,2]$. In fact, cooperative and coordinated control have attracted several research communities such as: biology [3]; artificial intelligence [4]; wireless sensor networks[ 5, 6]; control of mobile robot $[7,8]$; spacecraft $[9,10]$. The synchronized control of robot manipulators has been firstly presented in $[11,12]$. Subsequently, further research results on the synchronization of robot manipulators have been published $[13,14]$. It is recognized that the existing synchronization works as aforementioned are all for motion control. In this context, motion synchronization of multi agent systems has attracted much attention in various applications including the industrial assembling, automatic control of multi agent systems such as the control of robot manipulators $[15,16]$.

As an illustration, in [17] position synchronization of multiple motion axes has been studied. Motion synchronization has also been used in more complex mechanical systems especially the nonlinear robot systems. Furthermore, where flexibility and maneuverability are highly recommended [13, 18, 19], with the increasing complexity of evolved and specific applications, manipulability can't be fulfilled by a simple robot. For this reason, the use of cooperative schemes for multiple robots can present a better solution to realize more robust multi agent system controls, where each robots operates cooperatively, and receives feedbacks from each others to achieve a consolidated goal $[11,20]$.

Furthermore, most of the real systems are known by nonlinearities such as robotic field. For this reason, the formulation for robust control laws is required,in the sense that it is able to ensure the system stability and the robustness via external disturbances and parameters variations $[21,22]$. Otherwise, several control methods have been used to synchronize various complex systems such as: adaptative control [23, 24]; sliding mode control(SMC) $[25,26,27]$; neural networks [28] etc.

Among several dynamic behaviors, the synchronized motion control and the stability of complex nonlinear systems are considered between the most important research topics during several years [29, 30]. The mutual synchronization of robotic systems without time delay has been absolutely studied by several communities $[11,13]$. Nevertheless, and due to the importance of cooperative research using multiple agents and within its wide range applications, the control algorithm suffers from some disturbing factors that can't be neglected such as time delays communication, communication interruption, packet collisions etc. Time delay communication is commonly

*Marwa Fathallah, Sfax-Tunisia, fathallahmaroua@gmail.com 
known in biological and physical networks, owing to the finite velocity of communication as well as traffic congestions [31, 32, 33]. In multi-agent robotic systems, the time delay is only considered at the level of the information arriving to a robot and coming from sensors of its neighbors, while information from its own sensors is processed immediately.

The presence of this communication constraint makes the dynamical behaviours more complicated, can lead to undesirable transient response, reduces the performances of the networked systems or even the instability of the system [33, 34, 35]. Referring to the cross-coupling technique, several works have been suggested to improve the performances of the synchronization of multi axis motions $[36,37]$. Later on, [38] proposed a control algorithm in order to synchronize networked systems in the presence of time delays $[39,40]$. In the light of what was said, we present in this paper a synchronizing SMC algorithm for the control of a time delayed multi agent system in order to prove the effectiveness and the stability of the proposed approach.

\subsection{Contribution}

The subject of this paper is to realize the motion control of complex networked robotic systems in the presence of time delays.

Since the robot requires an interaction with its environment to achieve its goal, and in the absence of a suitable sensor, the robot remains blind. For this reason, and in order to reproduce human capacities for perception and action in robotic systems, researchers adopt the integration of data from a surveillance camera.

This camera is a great way to provide security to the target location. Nowadays, the surveillance cameras can also be set to be motion activated, recording footage when motion triggers them. Many range of cameras also includes outdoor security cameras and wifi cameras in order to effectively control the monitoring task.

Therefore, a second order sliding mode strategy has been considered and exhibited on a 3 degrees of freedom(3DOF) surveillance camera system, where we focus on the manipulative arm managing the camera movements.

Then, to guarantee the overall vision of the proposed framework, we combine a muli robot manipulators, where each robot must synchronize its movement with other teammates using the cross-coupling approach .

The main goal of this work is to realize a common and performant motion control task of multi-agent robot manipulators based on the cross coupled second order sliding mode approach design, by reducing the chattering impact, and achieving robust communication between agents which make the system stronger against disturbances, uncertainties, breakdowns and also able to compensate the existence of communication delay.

\section{Second Order Sliding mode con- troller}

\subsection{Preliminaries}

Sliding mode control is a robust nonlinear strategy [41, 46, 47]. Such Variable Structure Control (VSC) is considered as a discontinuous feedback approach where its design is divided into two parts: the reaching phase (system trajectories are forced to reach a specific surface in the state space then to remain on it) and the sliding phase (Figure 1 .

The Sliding Mode approach is developed using the Lyaponov Theory in order to ensure the convergence to the sliding surface $(s(x)=0)$ :

$$
S^{T} \dot{S}<0
$$

where the sliding surface $S$ is chosen as:

$$
S(x)=Y\left(x-x_{d}\right)
$$

in which: $x_{d}$ is the desired trajectory and $Y$ is a matrix chosen such away $x$ acheives $x_{d}$.

The structure of the proposed controller is composed of two terms:

$$
u=u_{e q}+\Delta u
$$

where $u_{e q}$ is the equivalent control which ensure the "reaching phase" and $\Delta u$ is the corrective term used to avoid all deviations from the sliding surface.

The expression of the equivalent term can be deduced from the following equation:

$$
\dot{S}=F(x)+G(x) u=0 \quad \mapsto \quad u_{e q}=-[G(x)]^{-1} F(x)
$$

where $F(x)$ and $G(x)$ are defined by the affine state equation of a nonlinear system $(\dot{x}=f(x)+g(x) u)$.

Moreover, the corrective term can be described as follows:

$$
\Delta u=-[G(x)]^{-1} W \operatorname{sign}(S)
$$

where $W$ is a definite positive matrix.

\subsection{Second Order Sliding Mode Control}

As it is mentioned above, this robust approach suffers from the undesirable chattering phenomenon [14, 34] induced by the corrective term $\Delta u$, whose impact is manifested by the existence of perturbing high switching frequencies in the control inputs [21, 42]. More precisely, this problem involves fast and sudden changing control signals which lead to low accuracy and even damage the mechanical parts. Therefore, several methods have been developed in order to overcome this annoying phenomenon [22].

In this context, the second order sliding mode approach presents an enhancement of the classical SMC by the introduction of a filtering action in the controller.

Such an action greatly reduces the major drawback of 


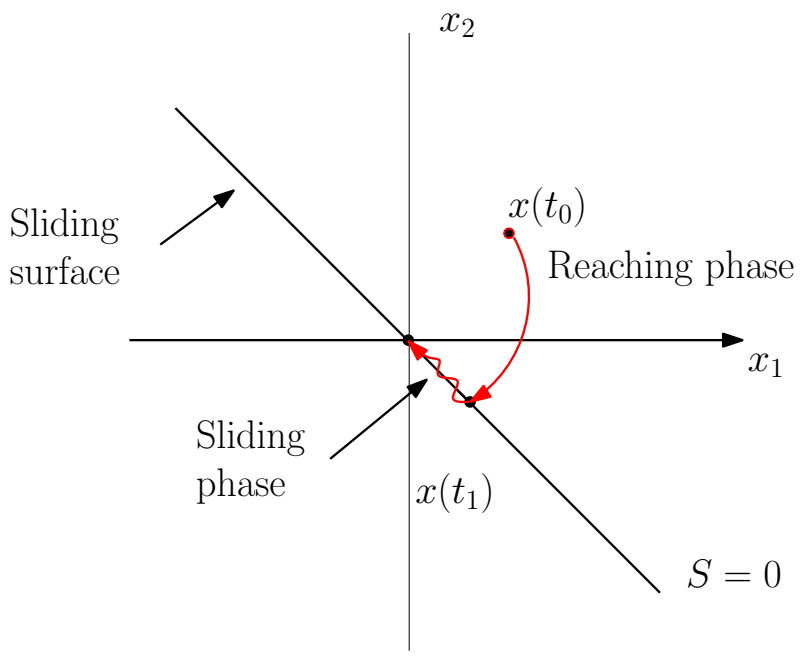

Figure 1: Phases of Sliding Mode Control

the simple SMC which is the chattering phenomenon. Hence, the second order SMC has been considered as the most useful among the high order SMC thanks to its relative simplicity of application, compared to the higher order controls [43, 44, 45].

Then, the sliding surface derivative of the second order SMC approach has been modified as follows :

$$
\dot{s_{i}}=\sigma_{i} \quad \mapsto \Delta u_{i}=[W g(x)]^{-1} \sigma_{i}
$$

The new description of the dynamic control behavior can be written as follows:

$$
\left\{\begin{aligned}
\dot{s_{i}} & =\sigma_{i} \\
\dot{\sigma_{i}} & =-a_{0} s_{i}-a_{1} \sigma_{i}+v_{i}
\end{aligned}\right.
$$

where $a_{0}, a_{1}$ are positive scalars and $v_{i}$ is a variable control of SMC. This study presents multi-inputmulti-output systems. Furthermore, the expression of $\dot{\sigma}_{i}$ can be deduced from the equality $A(p)=0$ of the following Hurwitz polynomial (which its roots have negative real parts) :

$$
A(p)=(p+\mu)^{2}
$$

where $\mu$ is a positive scalar.

In order to ensure the stability, the representation (7) can be reformulated as the condensed form:

$$
\dot{Z}_{i}=\phi Z_{i}+\Gamma v_{i}
$$

in which:

$$
\underbrace{\left(\begin{array}{c}
\dot{s_{i}} \\
\dot{\sigma_{i}}
\end{array}\right)}_{\dot{Z}_{i}}=\underbrace{\left(\begin{array}{cc}
0 & I \\
-\mu^{2} I & -2 \mu I
\end{array}\right)}_{\phi}\left(\begin{array}{l}
S_{i} \\
\sigma_{i}
\end{array}\right)+\underbrace{\left(\begin{array}{l}
0 \\
1
\end{array}\right)}_{\Gamma} v_{i}
$$

where 0 is the null matrix, $I$ is the identity matrix and the discontinuous term $v_{i}$ is given by .

$$
v_{i}=-Q \operatorname{sign}\left(\Gamma^{T} L S_{i}\right)
$$

where $Q=\left[q_{1}, q_{2}, \ldots, q_{n}\right]$ and $L$ are positive definite matrix.

\section{Mutual SMC synchronization al- gorithm}

\subsection{Mathematical model}

A robotic manipulator arm designed to be equipped with a surveillance camera system presents the adopted dynamic model of this study (Figure 2).

Using the Lagrangian formulation, the motion equation of a manipulator robot " $i$ " can be written as: [46]

$$
M_{i}\left(q_{i}\right) \ddot{q}_{i}+C_{i}\left(q_{i}, \dot{q}_{i}\right) \dot{q}_{i}+G_{i}\left(q_{i}\right)=\tau_{i}
$$

where:

- $q_{i}(t) \in \mathbb{R}^{n}$ is the measured articulation vector of the manipulator (joint position),

- $\dot{q}_{i} \in \mathbb{R}^{n}$ is the velocity vector,

- $\ddot{q}_{i} \in \mathbb{R}^{n}$ is the joint acceleration vector,

- $M_{i}\left(q_{i}\right) \in \mathbb{R}^{n \times n}$ is the symmetric uniformly bounded and positive definite inertia matrix,

- $C_{i}\left(q_{i}, \dot{q}_{i}\right) \dot{q}_{i} \in \mathbb{R}^{n}$ represents the vector expressing Coriolis and centrifugal forces,

- $G_{i}\left(q_{i}\right) \in \mathbb{R}^{n}$ is the vector of gravitational torques,

- $u=\tau_{i} \in \mathbb{R}^{n}$ denotes the control torque.

\subsection{Cross Coupling technique}

In this work, we take into consideration the synchronization of multiple robot manipulators. In this context, we propose decentralized control laws for $n$ robots manipulators for which each robot synchronizes its position with the other neighbor agents and track the same desired trajectory. Specifically, using the synchronization approach, manipulators are controlled in a synchronous manner so that the tracking errors and the synchronization errors converge 


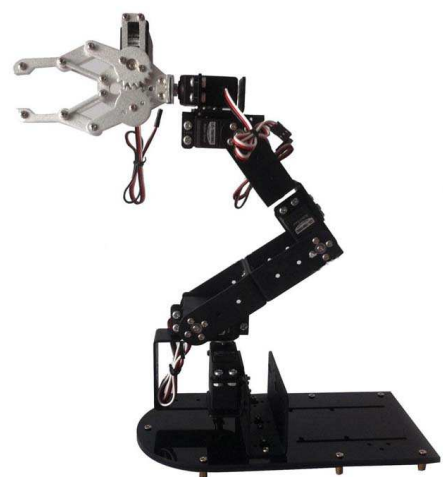

Figure 2: Example of 3DOF robot manipulator

to zero where the synchronization error is considered to be a differential position between coordinated agents. In order to achieve a coordinated control motion, an effective synchronization method namely the Cross Coupling concept is carried out where the whole multi-robot manipulators is used as a unique generalized system $[47,48,49]$.

The cross coupling design was firstly introduced by [2], then its concept has been used mainly for machine tools $[50,51]$. Later on, it has been applied in the robotic fields by [52]. The main idea of such a procedure is to create a global error of the model [36, 53].

In the light of what was said, the tracking error can be written as follows:

$$
\eta_{i}(t)=q_{i}(t)-q_{d}(t)
$$

where $q_{d}(t) \in \mathbb{R}^{n}$ denotes the desired position.

The vector $\eta_{i}$ will give insight on the joint positions convergence to the desired trajectory. The proposed cross coupling concept suggests a suitable synchronization error which is defined as follows:

$$
\begin{gathered}
\xi_{i}(t)=\sum_{j \neq i}^{p} \Lambda_{i j}\left[q_{i}(t)-q_{j}(t-\tau)\right] \\
=\sum \Lambda_{i j}\left(q_{i}(t)-q_{d}(t)\right)-\sum \Lambda_{i j}\left(q_{j}(t-\tau)-q_{d}(t-\tau)\right) \\
+\sum \Lambda_{i j}\left(q_{d}(t)-q_{d}(t-\tau)\right) \\
=\sum \Lambda_{i j} \eta_{i}-\sum \Lambda_{i j} \eta_{j}(t-\tau)+\sum \Lambda_{i j}\left[q_{d}(t)-q_{d}(t-\tau)\right]
\end{gathered}
$$

where $\Lambda_{i j}$ is a symetric positive definite matrix which reveals an idea about the communication quality between the $i^{\text {th }}$ and $j^{\text {th }}$ agents.

Thus, in order to attain a robust controller for multi robot systems, and to ensure a synchronous trajectory tracking in the presence of communication data delay, we define the following global error expression (for robot $i$ ):

$$
\varepsilon_{i}=\eta_{i}+\int_{t_{0}}^{t} \xi_{i}(\alpha) d \alpha
$$

where we note that this error expression includes both synchronization error and trajectory tracking error defined above.

Then, its derivative can be considered as follows:

$$
\dot{\varepsilon}_{i}=\dot{\eta}_{i}+\xi_{i}
$$

Let's define the sliding mode surface :

$$
s_{i}=\dot{\varepsilon}_{i}+\lambda_{i} \varepsilon_{i}
$$

where $\lambda_{i}>0$. Then, we obtain:

$$
\dot{\eta}_{i}=-\xi_{i}-\lambda_{i} \varepsilon_{i}+s_{i}
$$

Having in mind the expression of (14), we obtain the following expression:

$$
\begin{gathered}
\dot{\eta}_{i}=-\sum \Lambda_{i j} \eta_{i}+\sum \Lambda_{i j} \eta_{i}(t-\tau) \\
-\sum \Lambda_{i j}\left[q_{d}(t)-q_{d}(t-\tau)\right]-\lambda_{i} \varepsilon_{i}+s_{i}
\end{gathered}
$$

In order to simplify the previous expression, we define:

$d_{i}=\sum \Lambda_{i j}\left[q_{d}(t)-q_{d}(t-\tau)\right]$.

Thus:

$$
\dot{\eta}=A \eta+B \eta(t-\tau)+d-\Lambda \varepsilon+S
$$

where:

$A=\left(\begin{array}{ccc}-\sum \Lambda_{1 j} & \cdots & 0 \\ \vdots & \ddots & \vdots \\ 0 & \cdots & -\sum \Lambda_{n j}\end{array}\right), \quad \eta=\left[\begin{array}{c}\eta_{1} \\ \eta_{2} \\ \vdots \\ \eta_{n}\end{array}\right]$

$B=\left(\begin{array}{ccc}0 & \sum \Lambda_{12} \cdots & \sum \Lambda_{1 n} \\ \sum \Lambda_{21} & \sum \Lambda_{23} \cdots & \sum \Lambda_{2 n} \\ \vdots & \ddots & \vdots \\ \sum \Lambda_{n 1} & \cdots & 0\end{array}\right), S=\left(\begin{array}{c}s_{1} \\ s_{2} \\ \vdots \\ s_{n}\end{array}\right)$

$d=\left(\begin{array}{c}d_{1} \\ d_{2} \\ \vdots \\ d_{n}\end{array}\right), \Lambda=\left(\begin{array}{ccc}\lambda_{1} I & \ldots & 0 \\ \vdots & \ddots & \vdots \\ 0 & \cdots & \lambda_{n} I\end{array}\right)$

The equivalent control expression can be deduced from the equality $\dot{s}_{i}=0$ then, we obtain:

$u_{e q_{i}}=M_{i}\left[\ddot{q}_{d}-\dot{\xi}_{i}+\lambda_{i}\left(\xi_{i}+\dot{\eta}_{i}\right)\right]+C_{i}\left(q_{i}, \dot{q}_{i}\right) \dot{q}_{i}+g_{i}\left(q_{i}\right)$ 


\subsection{Theorem}

The control law:

$$
u=u_{e q_{i}}+\Delta u
$$

where: $u_{e q_{i}}$ is expressed in equation 21 and $\Delta u$ is given by:

$$
\Delta u=N R^{-1}[N \varepsilon-P \eta-\Lambda S-K \operatorname{sign} S]
$$

with $N$ is a diagonal, symmetric definite positive matrix which stabilities the proposed system composed by $n$ manipulators.

Remembering that for the Second Order Sliding Mode Control, the sliding surface becomes $\dot{s}_{i}=\sigma_{i}$ and taken into consideration equation (17), we obtain:

$\ddot{q}_{i}-\ddot{q}_{d}+\sum \Lambda_{i j}\left(\dot{q}_{i}-\dot{q}_{j}\right)+\lambda_{i}\left[\dot{q}_{i}-\dot{q}_{d}+\sum \Lambda_{i j}\left(q_{i}-q_{j}\right)\right]=\sigma_{i}$

Retaking the mathematical model equation 12 and substituting it in the previous equation gives:

$$
\begin{gathered}
\sigma_{i}=M_{i}^{-1}\left[\tau_{i}-C_{i}\left(q_{i}, \dot{q}_{i}\right) \dot{q}_{i}-G_{i}\left(q_{i}\right)\right] \\
+\sum \Lambda_{i j}\left(\dot{q}_{i}-\dot{q}_{j}\right)+\lambda_{i}\left[\dot{q}_{i}-\dot{q}_{d}+\sum \Lambda_{i j}\left(q_{i}-q_{j}\right)\right]-\ddot{q}_{d}
\end{gathered}
$$

And consequently,

$$
\begin{aligned}
\tau_{i}=C_{i}\left(q_{i}, \dot{q}_{i}\right) \dot{q}_{i}+G_{i}\left(q_{i}\right)+M_{i}\left[\ddot{q}_{d}-\sum \Lambda_{i j}\left(\dot{q}_{i}-\dot{q}_{j}\right)\right. \\
\left.-\lambda_{i}\left[\left(\dot{q}_{i}-\dot{q}_{d}\right)+\sum \Lambda_{i j}\left(q_{i}-q_{j}\right)\right]+\sigma_{i}\right]
\end{aligned}
$$

\section{Proof of the Stability Analysis}

In order to prove the stability of the considered multi agent system, a first Lyaponov function is chosen as follows:

$$
V_{1}=\eta^{T} P \eta
$$

Its derivative yields:

$$
\dot{V}_{1}=\eta^{T}\left(P A+A^{T} P\right) \eta+2 \eta^{T} P(B \eta(t-\tau)+d-\Lambda \varepsilon+S)
$$

The second function is expressed as:

$$
V_{2}=\int_{t-\tau}^{t} \eta^{T} H \eta d \alpha
$$

where:

$$
\dot{V}_{2}=\eta^{T} H \eta-\eta(t-\tau)^{T} H \eta(t-\tau)
$$

Then and in order to simplify the expression of $\dot{V}_{1}+\dot{V}_{2}$, we firstly compute the sub -equation:

$2 \eta^{T} P B \eta(t-\tau)-\eta(t-\tau)^{T} H \eta(t-\tau)$.

So, we denote that $\zeta_{1}=\rho_{1} \eta(t-\tau)-\frac{1}{\rho_{1}} D^{-1} B^{T} P \eta(t)$
Consequently:

$$
\begin{gathered}
\dot{V}_{1}+\dot{V}_{2}=\eta^{T}\left(P A+A^{T} P\right) \eta+2 \eta^{T} P(d-\Lambda \varepsilon+S) \\
+\eta^{T} H \eta-\zeta_{1} D \zeta_{1}+\rho_{1}^{2} \eta^{T} P B D^{-1} B^{T} P \eta \\
+\frac{1}{\rho_{1}^{2}} \eta(t-\tau)^{T} D \eta(t-\tau)-\eta(t-\tau)^{T} H \eta(t-\tau)
\end{gathered}
$$

After that, we regroup all terms of the previous equation:

$$
\begin{aligned}
\dot{V}_{1}+\dot{V}_{2} & =\eta^{T}\left(P A+A^{T} P+H+\rho_{1}^{2} P B D^{-1} B^{T} P\right) \eta \\
& -\zeta_{1}^{T} D \zeta_{1}-\eta(t-\tau)^{T}\left(H-\frac{1}{\rho_{1}^{2}} D\right)
\end{aligned}
$$

where $H$ and $D$ are positive definite matrix while $P$, $N$ are symetric positive matrix.

It's obvious from the previous equation that there are three terms relative to $d, \varepsilon$ and $S$ which should been developed. Consequently:

$$
2 \eta^{T} P d=-\zeta_{2}^{T} \zeta_{2}+\rho_{2}^{2} \eta^{T} \eta+\frac{1}{\rho_{2}^{2}} d^{T} P^{2} d
$$

in which:

$$
\left\{\begin{array}{c}
\zeta_{2}=\rho_{2} \eta-\frac{1}{\rho_{2}} P d \\
-2 \eta^{T} \Lambda \varepsilon=-\zeta_{3}^{T} \zeta_{3}+\rho_{3}^{2} \eta^{T} \eta \frac{1}{\rho_{3}^{2}} \varepsilon^{T} \Lambda^{T} \Lambda \varepsilon
\end{array}\right.
$$

where $\zeta_{3}=\rho_{3} \eta-\frac{1}{\rho_{3}} \Lambda \varepsilon$.

In the sequel, we introduce new terms $V_{3}$ and $V_{4}$ to complete the stability verification of the proposed synchronized control schemes such that:

$$
\left\{\begin{aligned}
V_{3} & =\varepsilon^{T} N \varepsilon \\
V_{4} & =S^{T} R S
\end{aligned}\right.
$$

The differentiation with respect of time gives:

$$
\left\{\begin{array}{l}
\dot{V}_{3}=2 \varepsilon^{T} N(s-\Lambda \varepsilon)=-\varepsilon^{T}\left(N \Lambda+\Lambda^{T} N\right) \varepsilon \\
-2 \varepsilon^{T} N s \\
\dot{V}_{4}=2 \dot{S}^{T} R S
\end{array}\right.
$$

Therefore, we develop the following terms:

$$
\left\{\begin{array}{l}
2 \eta^{T} P S=-\zeta_{4}^{T} \zeta_{4}+\rho_{4}^{2} \eta^{T} \eta+\frac{1}{\rho_{4}^{2}} S^{T} P^{2} S \\
-2 \varepsilon^{T} N S=-\zeta_{5}^{T} \zeta_{5}+\rho_{5}^{2} \varepsilon^{T} \varepsilon+\frac{1}{\rho_{5}^{2}} S^{T} N^{2} S
\end{array}\right.
$$

in which $\zeta_{4}=\rho_{4} \eta-\frac{1}{\rho_{4}} P S$ and $\zeta_{5}=\rho_{5} \varepsilon+\frac{1}{\rho_{5}} N S$.

Finally, the derivative of the global Lyaponov function

$V=V_{1}+V_{2}+V_{3}+V_{4}$ yields:

$$
\begin{aligned}
\dot{V} & =\eta^{T}\left(P A+A^{T} P+H+\rho_{1}^{2} P B D^{-1} B^{T} P+\rho_{2}^{2} I+\rho_{3}^{2} I\right. \\
& \left.+\rho_{4}^{2} I\right) \eta-\zeta_{1}^{T} D \zeta_{1}-\zeta_{2}^{T} \zeta_{2}-\zeta_{3}^{T} \zeta_{3}-\zeta_{4}^{T} \zeta_{4}-\zeta_{5}^{T} \zeta_{5} \\
& -\eta(t-\tau)^{T}\left(H-\frac{1}{\rho_{1}^{2}} D\right) \eta(t-\tau)-\varepsilon^{T}\left(N \Lambda+\Lambda^{T} N\right. \\
+ & \left.\rho_{5}^{2} I-\frac{1}{\rho_{3}^{2}} \Lambda^{T} \Lambda\right) \varepsilon+2 S^{T}(R \dot{S}-N \varepsilon+P \eta)+\frac{1}{\rho_{2}^{2}} d^{T} P^{2} d
\end{aligned}
$$



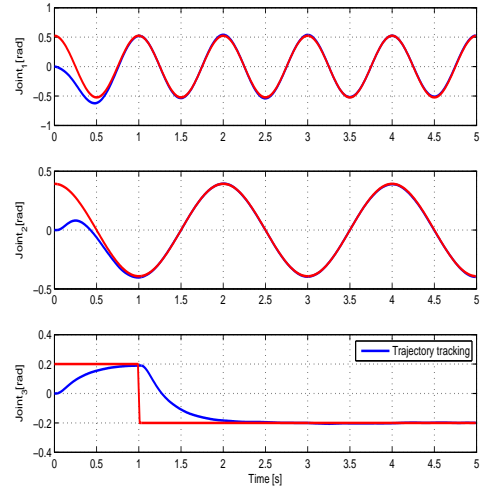

(a)
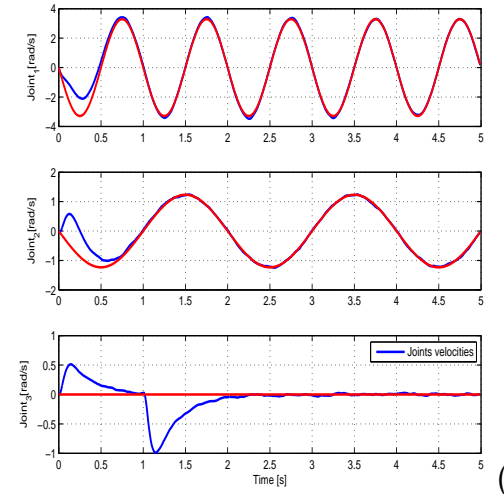

(b)

Figure 3: Simulation in the presence of external disturbances: (a) Positions evolutions in the presence of low measurement noises, (b) Velovities evolutions in the presence of low measurement noises
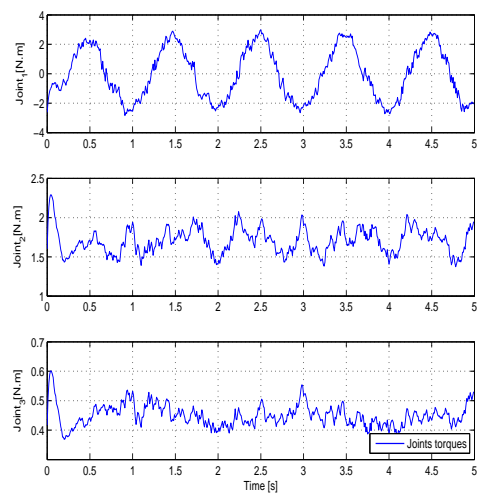
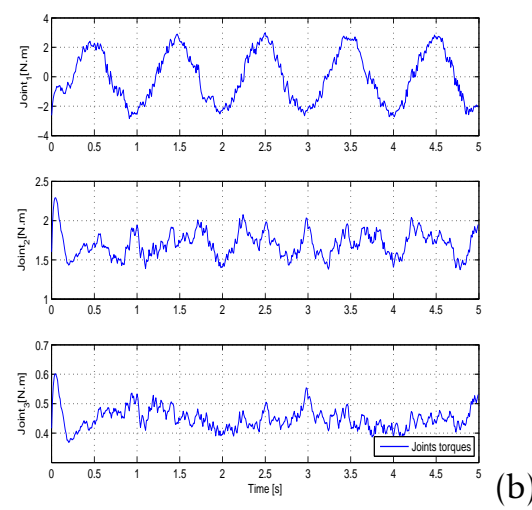

Figure 4: Simulation in the presence of external disturbances: (a) Torques evolutions of the SOSMC, (b) Measured torques evolutions of the SOSMC

Assume that $\dot{S}$ is defined as follows:

$$
\dot{S}=R^{-1}[-N \varepsilon-P \eta-\Lambda S-K \operatorname{sign} S]
$$

Hence, remember that $N, P, \Lambda, K$ are positive definite matrices, and assume that they verify the following conditions:

$$
\left\{\begin{array}{c}
H>\frac{1}{\rho^{2}} D \gg I \\
P A+A^{T} P+H+\rho_{1}^{2} P B D^{-1} B^{T} P+\left(\rho_{2}^{2}+\rho_{3}^{2}+\rho_{4}^{2}\right) I \\
N \Lambda+\Lambda^{T} N>\frac{1}{\rho_{3}^{2}} \Lambda^{T} \Lambda \\
2 S^{T}(R \dot{S}-N \varepsilon+P \eta)+\frac{1}{\rho_{2}^{2}} d^{T} P^{2} d=-2 S^{T} \Lambda S<0 \\
-2 K|S|+\frac{1}{\rho_{2}^{2}} d^{T} P^{2} d<0
\end{array}\right.
$$

The stability is confirmed if:

$\rho_{1}^{2} P B D^{-1} B^{T} P \ll P A+A^{T} P$ and the term $\frac{1}{\rho_{2}^{2}} d^{T} P^{2} d$ is considered as small bounded so that it can be neglected.

Consequently, we obtain:

$$
\dot{V}_{i} \leq 0
$$

Finally, this confirms the stability of the overall system.

\section{5 robustness via measurement noises effect}

In order to test the robustness of the proposed controller via uncertainties, an additive measurement errors have been introduced.

Then the measured state can be expressed as follows:

$$
x_{m}(t)=x(t)\left(1+b_{1}(t)\right)=x(t)+\Delta x(t)
$$

where $b_{1}$ is an additive bounded measure noise such that:

$\left\|b_{1}(t)\right\| \leq d_{1}$, and $d_{1}$ is a positive constant.

The level of the error effect has been varied from the lower impact to be gradually more intense, aiming to verify the controller's capacity to withstand such disturbances. At the beginning, low noise has been yield (we fluctuate the perturbation from 5 percent to 20 percent), we notice that the positions and the velocities evolutions still remain on the sliding surface which prove the robustness and the insensibility of the second order sliding mode control via disturbances (Figure 3). Then, the level has been increased (almost 30 percent), and in this case, the noise start to affect the tracking evolution of the system(Figure $5 b$ and Figure 6b).

It is obvious from Figure 4 that there is a similarity between the real torques and the measured ones, this 
Table 1: Joints parameters

\begin{tabular}{|c||c|c|c|}
\hline Articulation & Mass & Length & Initial position \\
\hline$q_{1}$ & $2.7132(\mathrm{~kg})$ & $0.2(\mathrm{~m})$ & $\pi / 6(\mathrm{rad})$ \\
\hline$q_{2}$ & $1.1446(\mathrm{~kg})$ & $0.15(\mathrm{~m})$ & $\pi / 4(\mathrm{rad})$ \\
\hline$q_{3}$ & $0.3392(\mathrm{~kg})$ & $0.1(\mathrm{~m})$ & $0.2(\mathrm{rad})$ \\
\hline
\end{tabular}

Table 2: Control parameters

\begin{tabular}{|c||c|}
\hline Control Parameters & Values \\
\hline$\Lambda_{i j}$ & 0.8 \\
\hline$\omega$ & 20 \\
\hline timedelay & 0.2 \\
\hline
\end{tabular}
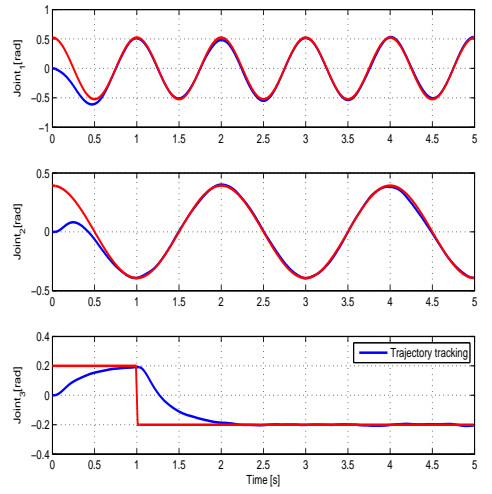
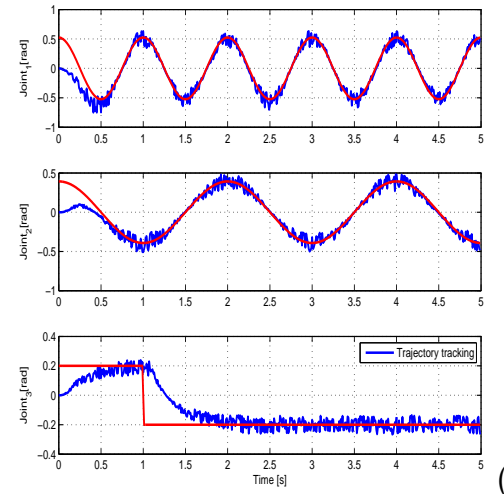

(b)

Figure 5: Simulations in the presence of external disturbances: (a) Positions evolutions in the presence of high measurement noises, (b) Measured Position evolutions in the presence of high measurement noises
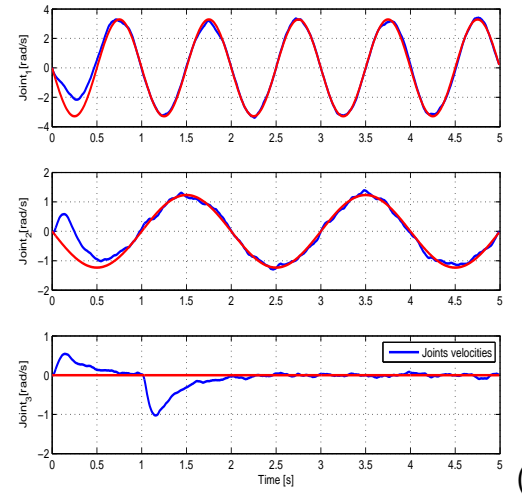

(a)
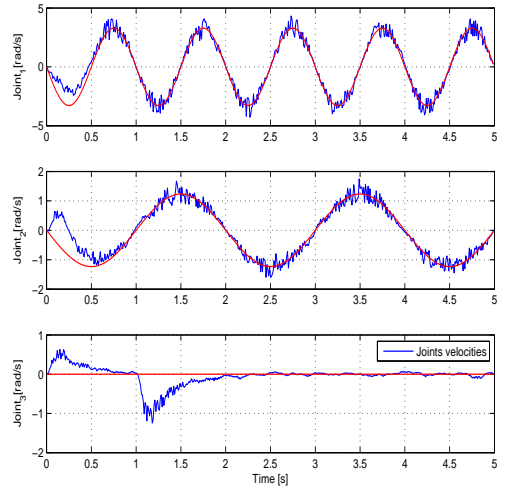

(b)

Figure 6: Simulations in the presence of external disturbances: (a) Velocities evolutions in the presence of high measurement noises, (b) Measured Velocities evolutions in the presence of high measurement noises

means that although the system suffers from external disturbances, the evolution of the applied torques do not record any notable increase, subsequently, no additional power consumption needed.

The aim of the comparison between real and measured simulations (Figure 5 and Figure 6) is to prove that the proposed controller exerts a satisfactory compensation action affecting the trajectory tracking and the velocity. This compensation becomes lower while increasing the disturbance effect, and it becomes unable to manage high imposed disruptions, i.e. starting from the presence of 30 present of errors applied to the system. Nevertheless, at this level the proposed controller has still resist to perturbations, and seen that the level of this disruption is relatively high, it can be then considered as a sufficient control, and proves its robustness via external disturbances. 

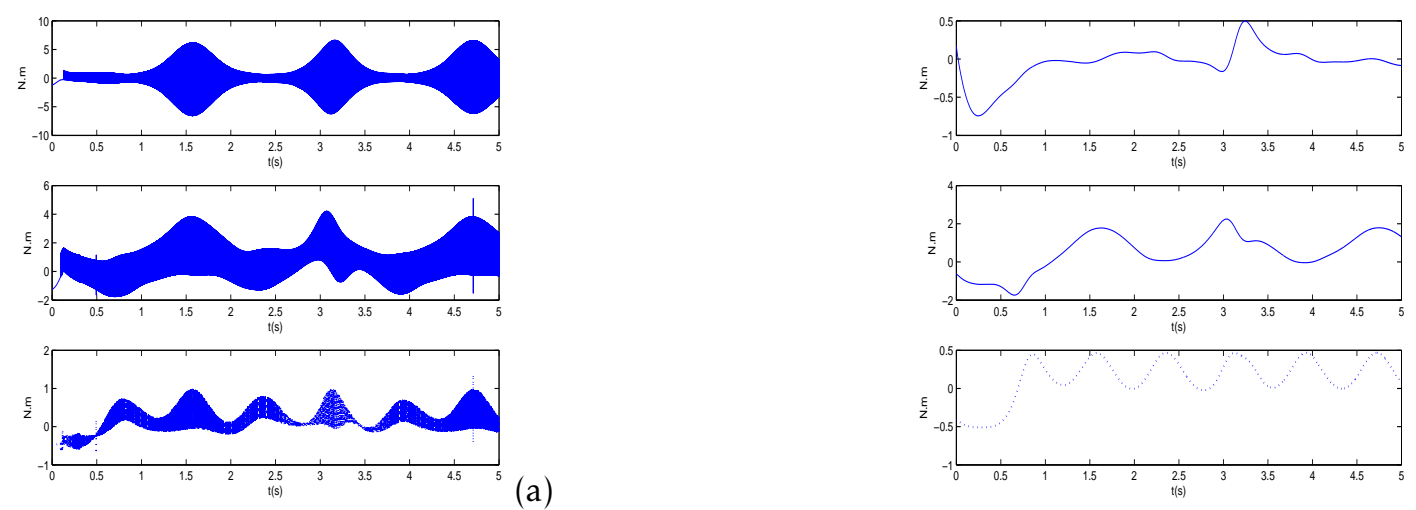

(b)

Figure 7: (a) The classical SMC torques evolutions, (b) The second order SMC torques evolutions
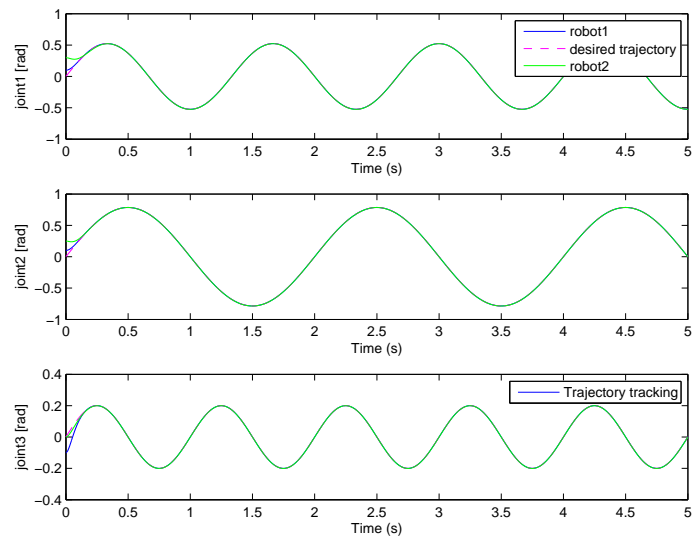

Figure 8: Motion Control and position synchronization
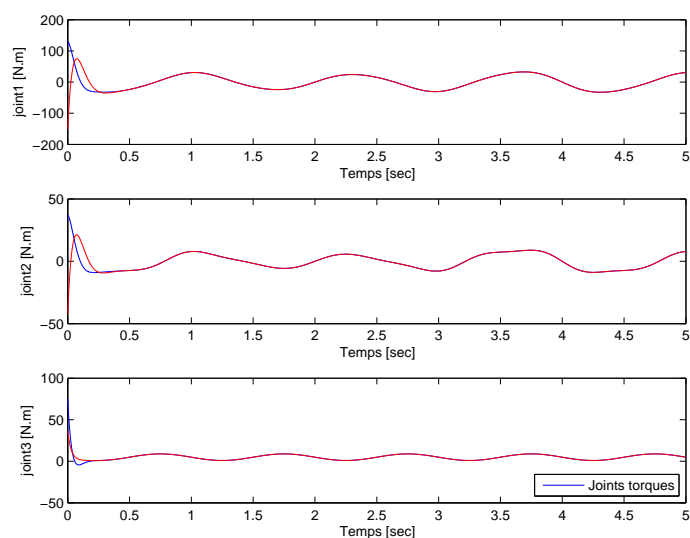

Figure 9: Evolution of the applied torques

\section{Simulation Results}

In this work, a second order SMC is applied to a networked multi-agent robotic system for a trajectory tracking control task. Indeed, the use of several cameras managed by manipulator robots and controlled by the proposed decentralized control law, allows the interaction of each robot with other agents in the networked system in order to make an overall vision about its environment. Thanks to the cross coupling concept, each agent is able to communicate and ex- change information with its neighbors. The desired trajectory is expressed by: 

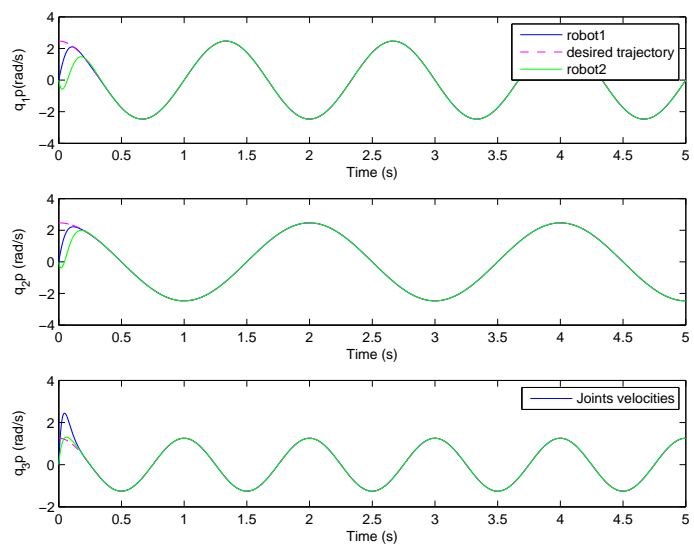

Figure 10: Velocity evolution of the synchronized robots

Parameters of the proposed system model used in simulation are illsutrated in Table 1 and control parameters values are given in Table 2.

Concerning the parameters $\mu_{i}$ and $\lambda_{i}$, they are defined as follows:

$$
\begin{gathered}
\lambda_{i}=2\left(\begin{array}{ccc}
1.125 & & 0 \\
& 1.45 & \\
0 & & 1.12
\end{array}\right) \\
\mu_{i}=\left(\begin{array}{lll}
3 & & 0 \\
& 4 & \\
0 & & 5
\end{array}\right)
\end{gathered}
$$

Simulation results show the robust synchronization and the smooth evolution in the trajectory tracking based on the second order SMC. The original SMC torques evolutions and the second order SMC ones are shown in Figure $7 a$ and Figure $7 b$ respectively.

It is obvious from the Figure $7 a$ that the classical sliding mode control suffers from the chattering phenomenon whose impact is reflected by the appearance of disrupting high switching frequencies (oscillations). Specifically, the problem consists of rapid and sudden changing control signals which lead to a low control accuracy. On the other side, Figure $7 b$ demonstrates that the second order SMC seems to be smooth and able to reduce the chattering phenomenon.

Besides, the presence of time delay between the cooperative robots is slightly reflected during the simulation as shown in Figure 8 and Figure 10 respectively. It may be said otherwise that the communication time delay between robots is clearly compensated and the position synchronization based on the cross coupling concept is obvious, predominately in the (Figure 8). The presence of undesirable phenomenon namely chattering in control torques of each agent is avoided in Figure 9

\section{Conclusion}

In this paper, the stability analysis of multi robot manipulator systems with a constant communication de- lay has been demonstrated. The main goal of this study is to compensate the delayed communication impact and to realize the synchronization between different robots of the system. So the proposed controlled system has succeeded to achieve a performing motion control task in the presence of loss of information during robot's communication, perturbations and also in the presence of delayed communication data. Simulation results show that the multi robots system can achieve the desired motion control task even with presence of a constant time delay.

\section{References}

1. Sarachik, P., Ragazzini, J. R., "A 2-dimensional feedback control system." Transactions of the American Institute of Electrical Engineers, Part II: Applications and Industry, 76(2), 55-61, 1957.

2. Koren, Y., "Cross-coupled biaxial computer control for manufacturing systems." Journal of Dynamic Systems, Measurement, and Control, 102(4), 265-272, 1980.

3. Beni, G., "The concept of cellular robotic system" Intelligent Control, Proceedings., IEEE International Symposium on, 102(4), 265-272, 1988.

4. Wei, H., Yong, W., Xuanqin, M., Yan, W., “A cooperative fuzzy control method for traffic lights." In Intelligent Transportation Systems, 2001. Proceedings. 2001 IEEE, 185-188, 2001.

5. Lucarelli, D., Wang, I. J. , “Decentralized synchronization protocols with nearest neighbor communication." In Proceedings of the 2nd international conference on Embedded networked sensor systems. ACM, 62-68, 2004.

6. Oh, M. K., Ma, X., Giannakis, G. B., Park, D. J., "Cooperative synchronization and channel estimation in wireless sensor networks." Journal of 
Communications and Networks, 7(3), 284-293, 2005.

7. Liu, S., Sun, D., Zhu, C., “Coordinated motion planning for multiple mobile robots along designed paths with formation requirement." IEEE/ASME transactions on mechatronics, 16(6), 1021-1031, 2011.

8. Shirkhodaie, A., "Supervised control of cooperative multi-agent robotic vehicles. In System Theory" Proceedings of the Thirty-Fourth Southeastern Symposium on. IEEE, 386-390, 2002.

9. Du, H., Li, S., Qian, C. , "Finite-time attitude tracking control of spacecraft with application to attitude synchronization." IEEE Transactions on Automatic Control, 56(11), 2711-2717, 2011.

10. Chung, S. J., Ahsun, U., Slotine, J. J. E., Miller, D. W., "Application of synchronization to cooperative control and formation flight of spacecraft" In proceedings of the AIAA Guidance, Navigation and Control Conference and Exhibit. Hilton Head, South Carolina, 2007.

11. Rodriguez-Angeles, A., Nijmeijer, H. , “ Mutual synchronization of robots via estimated state feedback: a cooperative approach." IEEE Transactions on Control Systems Technology, 12(4), 542-554, 2004.

12. Sun, D., Mills, J. K., "Adaptive synchronized control for coordination of two robot manipulators" In Robotics and Automation, 2002. Proceedings. ICRA'02. IEEE International Conference, 976-981, 2002.

13. Liu, Y. C., Chopra, N. , Park, D. J., “Controlled synchronization of heterogeneous robotic manipulators in the task space." IEEE Transactions on Robotics, 28(1), 286-275, 2012.

14. Zhao, D., Li, S., Gao, F., Zhu, Q., "Robust adaptive terminal sliding mode-based synchronised position control for multiple motion axes systems." IET Control Theory and Applications, 3(1), 136-150, 2009.

15. Lu, J., Yu, X., Chen, G., Cheng, D, "Characterizing the synchronizability of small-world dynamical networks." IEEE Transactions on Circuits and Systems I: Regular Papers , 51(1), 787796, 2004.

16. Tan, Yanxia, and Zhenkun Huang, "Synchronization of drive-response networks with delays on time scales." IEEE/CAA Journal of Automatica Sinica, 2016.

17. Sun, D., "Position synchronization of multiple motion axes with adaptive coupling control." Automatica, 39(6), 997-1005, 2003.
18. Li, Z., Ge, S. S., Adams, M., Wijesoma, W. S., "Robust adaptive control of uncertain force motion constrained nonholonomic mobile manipulators." Automatica, 44(3), 776-784, 2008.

19. Yang, C., Ganesh, G., Haddadin, S., Parusel, S., Albu-Schaeffer, A., Burdet, E., "Human-like adaptation of force and impedance in stable and unstable interactions." IEEE transactions on robotics, 27(5), 918-930, 2011.

20. Widyotriatmo, A., Hong, K. S., "Navigation function-based control of multiple wheeled vehicles." IEEE Transactions on Industrial Electronics, 58(5), 1896-1906, 2011.

21. Vaidyanathan, S., Azar, A. T., Hybrid synchronization of identical chaotic systems using sliding mode control and an application to Vaidyanathan chaotic systems, In Advances and applications in sliding mode control systems: Springer International Publishing, 549$569,2015$.

22. Shtessel, Y., Edwards, C., Fridman, L., and Levant, A., Introduction: Intuitive theory of sliding mode control. In Sliding Mode Control and Observation, Springer New York, 1-42, 2014.

23. Liao, Teh-Lu, Sheng-Hung Lin., "Adaptive control and synchronization of Lorenz systems." Journal of the Franklin Institute, 336(6), 925937, 1999.

24. Zhou, Qi, Chengwei Wu, Peng Shi., "Observerbased adaptive fuzzy tracking control of nonlinear systems with time delay and input saturation." Fuzzy Sets and Systems, 316, 49-68, 2016.

25. Yang, Shyi-Kae, Chieh-Li Chen, Her-Terng Yau, "Control of chaos in Lorenz system." Chaos, Solitons and Fractals, 13(4), 767-780, 2002.

26. Wang, J., Gao, Y., Qiu, J., and Ahn, C. K. , "Sliding mode control for non linear systems by Takagi Sugeno fuzzy model and delta operator approaches." IET Control Theory and Applications , 2016.

27. Yu, S., Chau, T. K., Fernando, T., Savkin, A. V., and $\mathrm{Iu}, \mathrm{H}$. H. C., "Novel Quasi-Decentralized SMC-Based Frequency and Voltage Stability Enhancement Strategies using Valve Position Control and FACTS Device." in IEEE Access, 2016.

28. Kazemy, Ali., "Complex network synchronization analysis with neural network nodes and time-delays." in Electrical Engineering (ICEE), 24th Iranian Conference on. IEEE, 2016.

29. Wu, Min, Yong He, and Jin-Hua She, Stability analysis and robust control of time-delay systems, Springer: Berlin, 2010. 
30. Lakshmanan, S., Prakash, M., Lim, C. P., Rakkiyappan, R., Balasubramaniam, P., and Nahavandi, S., "PSynchronization of an Inertial Neural Network With Time-Varying Delays and Its Application to Secure Communication" in IEEE Transactions on Neural Networks and Learning Systems, 2016.

31. Yao, J., Wang, H. N., Guan, Z. H., and Wang, H. O., "Synchronization of leader follower networks with coupling delays via variable structure control." Fuzzy Sets and Systems, 11(4), 407-410, 2009.

32. Yeganefar, N., "Definitions et analyse de stabilites pour les systemes retard non lineaires," Ph.D Thesis, University of Science and Technology of Lille-Lille I, 2006.

33. Wu, M., He, Y., and She, J. H., Stability analysis and robust control of time-delay systems, Berlin: Springer, 2010.

34. Jribi, A., Abdelhedi, F., Bouteraa, Y., and Derbel, N., Kinematics and a Comparison Between Two SM Control Strategies for a 5DOF Serial Robot for Tele-Echography, In Applications of Sliding Mode Control, 157-174, 2009.

35. Abdelhedi F., Derbel N., "Adaptive Second order sliding mode control based cross coupling concept for synchronization of robotic systems", Advances in Systems, Signals and Devices, Issues on Systems, Analysis and Automatic Control, 2016.

36. Srinivasan, K., Kulkarni, P. K., "Cross-coupled control of biaxial feed drive servomechanisms." Journal of dynamic systems measurement and control, 112(2), 225-232, 1990.

37. Koren, Y., Lo, C. C., "Advanced controllers for feed drives." CIRP Annals-Manufacturing Technology, 41(2), 689-698, 1992.

38. Liu, Yen-Chen, and Nikhil Chopra, "Synchronization of networked mechanical systems with communication delays and human input." Journal of Dynamic Systems, Measurement, and Control, 135(4), 041004, 2013.

39. Sun, Dong., Synchronization and control of multiagent systems, CRC Press, 2016.

40. Wu, Z. G., Park, J. H., Su, H., Chu, J., “Discontinuous Lyapunov functional approach to synchronization of time-delay neural networks using sampled-data." Nonlinear Dynamics, 69(4), 2021-2030, 2012.

41. Shafiei, S. E., Sliding mode control of robot manipulators via intelligent approaches, INTECH Open Access Publisher, 2010.
42. Levant, A., "Chattering analysis.” IEEE Transactions on Automatic Control, 55(6), 1380-1389, 2010 .

43. Bouaziz, F. A., Bouteraa, Y., and Medhaffar, H., "Distributed sliding mode control of cooperative robotic manipulators" In 10th International Multi-Conference on Systems, Signals and Devices, 2013.

44. Ferrara, A., Magnani, L., "Motion control of rigid robot manipulators via first and second order sliding modes." Journal of Intelligent and Robotic Systems, 48(1), 23-36, 2007.

45. Zhao, Dongya, and Quanmin Zhu., "Position synchronised control of multiple robotic manipulators based on integral sliding mode." International Journal of Systems Science, 45(3), 556570, 2014.

46. Fathallah, M., Abdelhedi, F., Derbel, N., " A surveillance camera algorithm based on the sliding mode approach with measurement's disturbance" In 13th International Multi-Conference on Systems, Signals and Devices, 2016.

47. Abdelhedi, F., Jribi, A., and Derbel, N. , " Robust SMC for synchronization of networked time delay robotic systems" In 28th International Conference on Modelling, Identification and Control (ICMIC), 2016.

48. Nouri, A. S., Bouazi, F. A., and Derbel, N., On the Sliding Control, In Applications of Sliding Mode Control Springer Singapore, 2017.

49. Bouaziz, F. A., Bouteraa, Y., and Derbel, N., "Control energy comparison between 1st and 2nd order sliding mode approach with application to a SCARA robot" In 13th International Multi-Conference on Systems, Signals and Devices, 2016.

50. Koren, Y., "Cross-coupled biaxial computer control for manufacturing systems." Journal of Dynamic Systems, Measurement, and Control, 102(4), 265-272, 1980.

51. Srinivasan, K., Kulkarni, P. K., "Cross-coupled control of biaxial feed drive servomechanisms." Journal of dynamic systems, measurement, and control, 112(2), 225-232, 1990.

52. Feng, L., Koren, Y., Borenstein, J., "Crosscoupling motion controller for mobile robots." IEEE control systems, 13(6), 35-43, 1993.

53. Abdelhedi, F., Bouteraa, Y. and Derbel N., Second Order Sliding Mode Based Synchronization Control for Cooperative Robot Manipulators, Advances and Applications in Nonlinear Control Systems Springer International Publishing, 2016. 